\title{
Perfil de valores de estudiantes de carreras de salud del sur de Chile
}

\author{
Alex Véliz Burgos ${ }^{1}$, Anita Dörner Paris², Edgardo Gonzáles Sierra², Miguel Ripoll Novales ${ }^{3}$
}

\section{RESUMEN}

Objetivo: Las instituciones de educación superior deben implementar en sus planes curriculares estrategias que posibiliten decisiones guiadas por principios y valores propios de cada profesión. El objetivo del estudio fue determinar el perfil de valores expresados por estudiantes de la salud en el sur de Chile.

Materiales y métodos: Se trata de un estudio cuantitativo de corte transversal, descriptivo, con muestra intencionada de 242 ( $M=20,1$ años) estudiantes de enfermería, psicología y kinesiología a quienes se aplicó el cuestionario Perfil de Valores Personales de Schwartz. Se utilizó el programa SPSS versión 20.0 para realizar un análisis de conglomerado jerárquico y verificar las dimensiones de valores asociadas entre sí, y se aplicó la prueba ANOVA de una vía con pruebas post hoc para verificar diferencias de medias en los puntajes de las subescalas de valores.

Resultados: Se identificaron dos grandes conglomerados homogéneos: uno conformado por las dimensiones conformidad, seguridad, realización, tradición y benevolencia, y otro conformado por hedonismo y estímulo. En el análisis de medias, se observan diferencias estadísticamente significativas entre los grupos en las dimensiones universalismo, poder, autodirección, benevolencia, conformidad, tradición y realización $(p<0,01)$. Las diferencias de medias más relevantes se encuentran entre las carreras de psicología y enfermería. En las dimensiones poder, conformidad y tradición, los estudiantes de kinesiología y enfermería presentan medias más altas que los de psicología $(p<0,01)$.

Conclusiones: Los resultados muestran un perfil valórico de los estudiantes de la salud. El siguiente paso debería ser la comparación del perfil resultante con el perfil valórico de egreso de cada disciplina, para favorecer el fortalecimiento de las dimensiones que cada carrera considera importantes para el desempeño profesional.

Palabras clave: Educación en salud; valores sociales; educación superior; enfermería; psicología; kinesiología.

\section{Values profile of health career students from Southern Chile}

\author{
ABSTRACT
}

Objective: Higher education institutions must include curricular strategies that enable students to make decisions according to the values and principles related to their professions. The objective of this study was to determine the values profile expressed by health career students from Southern Chile.

Materials and methods: This is a quantitative cross-sectional descriptive study with a purposive sample of $242(\mathrm{M}=20.1$ years old) nursing, psychology and kinesiology students to whom the Schwartz Value Survey was applied. Software SPSS 20.0 was used to perform a hierarchical clustering analysis and verify the values dimensions associated with each other. One-way ANOVA with post-hoc tests were conducted to verify the means differences among the scores of the values subscales.

Results: Two large homogeneous clusters of values were identified. One consisted of the dimensions of Conformity, Security, Achievement, Tradition and Benevolence, and the other one of the dimensions of Hedonism and Stimulation. During the analysis of means, significant statistical differences were observed between the groups in the dimensions of Universalism, Power, Self-Direction, Benevolence, Conformity, Tradition and Achievement $(p<0.01)$. The most outstanding means differences were those between psychology and nursing students. In the dimensions of Power, Conformity and Tradition, kinesiology and nursing students showed higher means than psychology students $(p<0.01)$.

Conclusions: The results reveal health career students' values profile. The next step should be to compare those profiles with the values profile of graduates from each discipline, in order to contribute to the strengthening of the dimensions which students from each career consider important for their professional performance.

Keywords: Health education; social values; higher education; nursing; psychology; kinesiology.

1. Académico Departamento de Ciencias Sociales, Universidad de Los Lagos. Chile.

2. Académica Departamento de Salud, Universidad de Los Lagos. Chile.

3. Académico Departamento de Ciencias Sociales, Universidad de Los Lagos. Chile. 


\section{INTRODUCCIÓN}

La expansión de la matrícula en Chile ha modificado el mapa de las instituciones de educación superior, pasando de 172.996 estudiantes matriculados en 1983 a 1.215 .413 en el año 2014(1). Un porcentaje importante de estos estudiantes provienen de sectores vulnerables. Esto implica que las instituciones formadoras, especialmente aquellas de carácter estatal deben desarrollar y garantizar un acceso con equidad, generar estrategias de nivelación y flexibilizar los currículos para el nuevo perfil de estudiantes que ingresa, evitar la deserción de estudiantes especialmente en los primeros años y establecer un perfil docente idóneo ${ }^{(2,3)}$.

Entre los desafíos está la generación de perfiles específicos de los estudiantes que se incorporan a las aulas con el fin de establecer las adecuaciones necesarias en este escenario masificado. Estas adecuaciones debieran realizarse tanto en términos pedagógicos como en aquellos factores psicosociales que afectan el desempeño y éxito académico de manera permanente de los estudiantes ${ }^{(3,4,5)}$.

En este sentido, la universidad debe asumir el valor social y público que tiene desde la formación integral de la persona, la complejidad del conocimiento y construcción de los procesos de elevación moral y cultural de la humanidad $^{(6)}$.

Estos estudiantes universitarios se enfrentan al desafío de desarrollar mayores niveles de autonomía, nuevas relaciones sociales, mantener un promedio de calificaciones mínimo, gestión del tiempo personal, el traslado de su lugar de residencia y adaptación a la vida universitaria.

Surge aquí un desafío importante para las instituciones formadoras, exigiendo no sólo establecer planes curriculares fundamentados en componentes científicos y técnicos sino implementar estrategias que posibiliten la formación de fundamentos éticos aplicados a la disciplina con el fin de formar profesionales que den respuestas de manera integral a las necesidades en salud que presentan los usuarios. Esto es lo que podríamos denominar un cuidado integral y holístico.

Para ello, será preciso conocer los valores profesionales que se forman en las instituciones de educación superior, pues a través de un sistema adecuado de valores, se garantiza un trato mejor a las personas usuarias de los servicios de los/las profesionales de salud. Por ello, la identificación de los valores que sean comunes a lo esencial de la identidad de cualquier profesional de la salud y la estimación e incorporación de los mismos por las nuevas generaciones, requieren del diseño y aplicación de métodos que permitan conocer cuáles son los valores estimados y hasta qué punto pueden estos orientar hacia los fines de cada profesión.

Respecto a este aspecto el psicólogo Shalom Schwartz, propone que los valores son metas deseables y variables en su grado de importancia y que orientan la vida y las conductas de una persona o de una institución. En un estudio desarrollado en más de 40 países ${ }^{(7)}$ se encontró que había diez valores que subyacían a todas las culturas estudiadas. Todos ellos se agrupan en dos bipolaridades (dimensiones) que contrastan, por un lado, la trascendencia de los intereses personales en función de los colectivos (Autotrascendencia) y, en el otro extremo, aquellas personas que priorizan sus intereses propios (Autopromoción), mientras que por otro lado, algunas personas aprecian la seguridad y el orden (Conservación) y contrariamente, otras valoran la independencia de acciones y pensamientos (Apertura al cambio). A su vez, todas estas polaridades definen intereses. Los intereses están más relacionados con los aspectos sociales y culturales (más generales). Pueden definirse intereses colectivistas (los valores que predominan en una sociedad, tienen que ver con atender a los intereses de la comunidad entera), individualistas (se valoran aquellos aspectos que tienen que ver con el desarrollo y éxito personales) o mixtos. Para Schwartz ${ }^{(8)}$ los valores representan las respuestas que las personas y los grupos deben dar a tres requisitos universales: (a) las necesidades de los individuos en su condición de seres biológicos, (b) la coordinación de las acciones sociales y (c) el funcionamiento correcto y la supervivencia de los grupos.

Los diez valores o tipos motivacionales reconocidos por Schwartz ${ }^{(8)}$ son los siguientes:

1. Poder: se refiere al interés por lograr poder social, autoridad, riqueza.

2. Logro/realización: se refiere a la búsqueda del éxito personal, poniendo en juego competencias que son socialmente aceptables.

3. Hedonismo: importan el placer y la gratificación personal, poder disfrutar de la vida.

4. Estimulación: importan la novedad y los desafíos.

5. Autodirección: se refiere al interés por poder pensar con independencia, tener libertad de acción y exploración, poder ser creativo.

6. Universalismo: importan la tolerancia y la justicia social, la protección del medio ambiente.

7. Benevolencia: importa preservar e intensificar el bienestar de las personas, la honestidad y la ausencia de rencor.

8. Tradición: interesan el respeto y el compromiso con las costumbres y la cultura tradicional, como así también con la religiosa.

9. Conformidad: se valoran las normas sociales y se evitan los comportamientos que puedan herir o molestar a los demás, se respeta a los mayores.

10. Seguridad: interesan la seguridad, armonía y estabilidad sociales y personales.

En América Latina se han realizado numerosos estudios a partir de esta teoría y, más específicamente en Chile, este modelo ha sido estudiado en diversos contextos y con distintos fines, contándose en la actualidad con instrumentos para la medición de los diez tipos valóricos, que han presentado evidencias apropiadas de validez y confiabilidad en población chilena ${ }^{(9)}$, permitiendo evaluar empíricamente el ajuste del modelo a los datos. El Cuestionario de Valores de Schwartz ${ }^{(10,11)}$.

Sin embargo, son escasos los estudios que ponen en juego los argumentos teóricos de este modelo en relación a la población de jóvenes estudiantes universitarios. La relevancia del estudio de este grupo socio demográfico radica en que, tal como plantean Carrasco y Osses ${ }^{(12)}$, es en la juventud -aquel período comprendido entre los $18 \mathrm{y}$ los 25 años- cuando se inicia el período de consolidación definitiva de los valores personales adquiridos en la infancia, que perdurarán y se mantendrán más menos constantes a lo largo de toda la vida. Y en ese período la 
universidad juega un rol especial, constituyéndose como un espacio educacional que cumple una doble función: por un lado, transmite los conocimientos, disciplinas y programas; y por otro, forma a los jóvenes y se convierte en el espacio donde se transmite la cultura, las actitudes, los hábitos e incluso también los valores ${ }^{(13)}$.

Estos resultados apoyan el carácter transcultural de la teoría, ya que se ha reportado un extendido consenso en el orden jerárquico de los tipos valóricos que pudiese ser explicado por la función adaptativa de satisfacción de requerimientos básicos para la preservación de la naturaleza humana y el funcionamiento social que los valores de universalismo y benevolencia implican ${ }^{(7)}$.

Por otra parte, los valores que los estudiantes universitarios jerarquizaron como menos importantes fueron poder y logro, que corresponden a valores relacionados a Autopromoción. Esta dimensión enfatiza la satisfacción de los intereses personales, aún a costa de los demás ${ }^{(14)}$, tanto en términos de aumento de estatus, control o dominio sobre personas o recursos, como en términos de éxito personal, mediante demostraciones de competencia según distintos criterios sociales ${ }^{(9)}$.

En función de lo anterior, se plantea como objetivo del estudio determinar el perfil de valores expresados por estudiantes de la salud en el sur de Chile.

\section{MATERIALES Y MÉTODOS}

\section{Diseño}

El estudio es de tipo descriptivo de corte transversal, no experimental pretendiendo mostrar el perfil valórico que presentan los estudiantes de salud.

La aplicación del instrumento se ha realizado de la manera menos intrusiva posible. Se puede señalar que los resultados de un estudio son válidos ecológicamente porque las conjeturas que se obtendrán tienen en cuenta el contexto donde se producen los fenómenos, en este caso las aulas universitarias.

\section{Participantes}

La muestra se obtuvo a través de un muestreo incidental y correspondió a estudiantes que cursan carreras de salud en una Universidad de la ciudad de Puerto Montt, Chile y que contestaron voluntariamente un cuadernillo que incluía datos sociodemográficos y el cuestionario de estudio.

La muestra final de investigación está formada por 242 estudiantes $(M=20,1, \quad D T=3,5$ años), pertenecientes a las carreras de Enfermería, Kinesiología y Psicología, se procuró contar con un número equivalente de cada carrera (Tabla 1).

Tabla 1. Sensibilidad, especificidad, valor predictivo positivo y valor predictivo negativo de los índices de PLVI

$\begin{array}{crrr}\text { Carreras } & \text { N } & \text { M } & \text { DT } \\ \text { Psicología } & 86 & 20 & 2,31 \\ \text { Enfermería } & 87 & 20 & 3,87 \\ \text { Kinesiología } & 69 & 19,28 & 2,04 \\ \text { Total } & 242 & 20,1 & 3,5\end{array}$

\section{Instrumentos de medida}

Se utilizaron dos instrumentos, ambos autoadministrados. El primero, una encuesta de elaboración propia que recogió datos biosociodemográficos y curriculares. El segundo instrumento utilizado fue "Perfil de Valores Personales", (Portrait Values Questionnaire, Schwartz) ${ }^{(15)}$, que es una escala que evalúa valores humanos. Este instrumento consta de dos dimensiones, distribuidas en dos listas de 31 valores centrales y 27 secundarios, medidos en escala de -1 a 7, en orden de importancia, donde el -1: corresponde a lo opuesto a mis valores y 7: está asociado a la mayor importancia que le asigna el estudiante.

Los diez valores o tipos motivacionales que muestra éste instrumento son los siguientes: poder, logro, hedonismo, estimulación, autodirección, universalismo, benevolencia, tradición, conformidad y seguridad.

\section{Procedimiento}

Se toma contacto con los directivos de cada carrera involucrada, se solicita apoyo para realizar el estudio y se explican los objetivos generales de la investigación.
Se fija un itinerario a cumplir con cada carrera en días y horarios que las jefaturas proporcionan a fin de no interrumpir la aplicación de los instrumentos. Se acude a cada aula, se explica el objetivo general del estudio, y se solicita que cada estudiante responda voluntariamente el cuadernillo. Sólo responden los sujetos presentes que voluntariamente desean hacerlo y que acceden a firmar documento de consentimiento informado. Los cuestionarios respondidos por los estudiantes fueron codificadas para asegurar anonimato y confidencialidad de la información. Los aspectos éticos de la investigación fueron resguardados según la normativa oficial vigente y de acuerdo a los principios planteados por Emanuel ${ }^{(16)}$. Durante la aplicación del instrumento se responden las dudas de los estudiantes respecto a los ítems y las instrucciones de respuesta. La duración promedio de la aplicación es de 30 minutos. Los datos obtenidos son codificados y analizados en SPSS 20.0. Posteriormente se realizó un análisis de conglomerado jerárquico para verificar las dimensiones de valores asociadas entre sí y en segundo lugar la prueba anova de una vía con pruebas post hoc HSD Tukey para verificar diferencias de medias de los puntajes de las subescalas de valores. 


\section{RESULTADOS}

A continuación se presentan los resultados del estudio.

En primer lugar, se verificó la confiabilidad de las dimensiones del cuestionario a través del estadístico Alfa de Cronbach. Como se observa en la tabla 2, la confiabilidad de las subescalas va de 0,467 a 0,821, siendo las confiabilidades más bajas aquellas dimensiones que presentan un menor número de ítems Seguridad (3 ítems) versus las dimensiones con mayor número de ítems (Universalismo, con 10 ítems).

Tabla 2. Confiabilidad de las dimensiones la escala de valores de Schwartz

$\begin{array}{lc}\text { Dimensión } & \text { Valor alfa } \\ \text { Universalismo } & 0,821 \\ \text { Poder } & 0,712 \\ \text { Hedonismo } & 0,468 \\ \text { Autodirección } & 0,655 \\ \text { Benevolencia } & 0,665 \\ \text { Conformidad } & 0,675 \\ \text { Estímulo } & 0,607 \\ \text { Seguridad } & 0,467 \\ \text { Tradición } & 0,599 \\ \text { Realización } & 0,686\end{array}$

Dendrograma que utiliza una vinculación media (entre grupos) Combinación de conglomerados de distancia re-escalado

Conformidad
Seguridad
Realización
Tradición
Benevolencia
Autodirección
Hedonismo
Estimulo
Poder

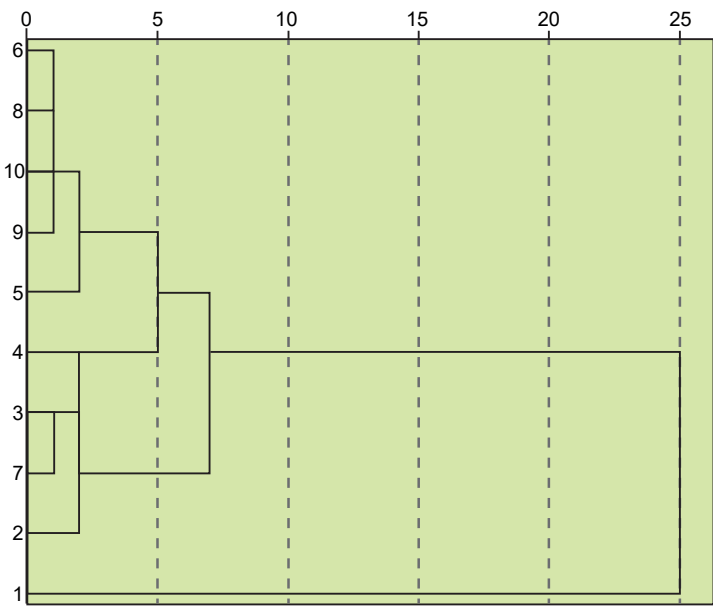

Figura 1. Dendograma estructuración de dimensiones de la Escala de Valores de Schwartz en estudiantes de salud del sur de Chile

Al realizar un análisis se observa que existen dos grandes conglomerados homogéneos en la muestra total de estudiantes. El primer conglomerado conformado por las dimensiones conformidad, seguridad, realización y tradición y un segundo conglomerado conformado por las dimensiones hedonismo y estímulo. Llama la atención que universalismo no se relaciona con otras dimensiones, sino que se presenta como una dimensión independiente. 
Tabla 3. Prueba anova de dimensiones de la escala de Valores de Schwartz entre estudiantes de psicología, kinesiología y enfermería

$\begin{array}{ll}\text { Dimensión } & \text { Sig. } \\ \text { Universalismo } & 0,007 \\ \text { Poder } & 0,010 \\ \text { Hedonismo } & 0,823 \\ \text { Autodirección } & 0,026 \\ \text { Benevolencia } & 0,003 \\ \text { Conformidad } & 0,000 \\ \text { Estímulo } & 0,529 \\ \text { Seguridad } & 0,061 \\ \text { Tradición } & 0,000 \\ \text { Realización } & 0,001\end{array}$

Los resultados de la prueba anova que se observan en la tabla 3 . indican que existen diferencias estadísticamente significativas $(p<0,05)$ en las dimensiones universalismo, poder, autodireccion, benevolencia, conformidad tradición y realización entre las carreras de enfermería, psicología y kinesiología.

En la tabla 4, por su parte se observan las medias de cada dimensión del cuestionario de valores. Si bien se aprecia que las medias de la carrera de enfermería son las más altas, cuando se verifica estas diferencias en las pruebas post hoc (tabla 5) se observa además que las medias que resultan con mayores diferencias estadísticamente significativas corresponden a la carrera de enfermería y psicología. Solamente en las dimensiones poder, conformidad y tradición, kinesiología y enfermería conforman un conglomerado diferenciado de psicología. Por otra parte, en universalismo, psicología y kinesiología conforman un grupo homogéneo.

Tabla 4. Medias de desempeño en la escala total y por carrera en las dimensiones de la escala de Valores de Schwartz

$\begin{array}{lrrrr}\text { Dimensión } & \text { Total } & \text { Psicología } & \text { Kinesiología } & \text { Enfermería } \\ \text { Universalismo } & 5,36 & 5,19 & 5,22 & 5,64 \\ \text { Poder } & 3,06 & 2,69 & 3,23 & 3,28 \\ \text { Hedonismo } & 4,58 & 4,57 & 4,5 & 4,66 \\ \text { Autodirección } & 6,43 & 6,25 & 6,35 & 6,66 \\ \text { Benevolencia } & 4,34 & 4,12 & 4,32 & 4,56 \\ \text { Conformidad } & 5,33 & 4,93 & 5,44 & 4,65 \\ \text { Estímulo } & 4,53 & 4,51 & 4,39 & 5,65 \\ \text { Seguridad } & 5,04 & 4,86 & 5,1 & 4,17 \\ \text { Tradición } & 4,48 & 4,07 & 4,7 & 4,73 \\ \text { Realización } & 4,94 & 4,65 & 4,89 & 5,26\end{array}$

Tabla 5. Diferencias de medias significativas por carrera en las dimensiones de la escala de Valores de Schwartz.con prueba HSD Tukey

\begin{tabular}{|c|c|c|}
\hline Dimensión & Carrera con media menor & Carrera con media Mayor \\
\hline Universalismo & $\begin{array}{l}\text { Psicología } \\
\text { Kinesiología }\end{array}$ & Enfermería \\
\hline Poder & Psicología & $\begin{array}{l}\text { Enfermería } \\
\text { Kinesiología }\end{array}$ \\
\hline Autodirección & Psicología & Enfermería \\
\hline Benevolencia & Psicología & Enfermería \\
\hline Conformidad & Psicología & $\begin{array}{l}\text { Enfermería } \\
\text { Kinesiología }\end{array}$ \\
\hline Tradición & Psicología & $\begin{array}{l}\text { Enfermería } \\
\text { Kinesiología }\end{array}$ \\
\hline Realización & Psicología & \\
\hline
\end{tabular}




\section{DISCUSIÓN}

En primer lugar se comentan datos de fiabilidad del estudio. Si bien se esperaba que los niveles de confiabilidad sean superiores a, $70^{(17}$, existen diversos autores que plantean que en estudios con propósitos de investigación no clínicos estos porcentajes pueden variar y ser inferiores incluso a, 60, todo dependerá de la utilización que se dé al instrumento ${ }^{(18,19)}$. Por otra parte, para verificar la dimensionalidad del instrumento, este debería ser sometido a un análisis factorial confirmatorio, constituyéndose este análisis en una tarea a abordar en un estudio posterior.

Respecto a los resultados de valores, se encuentra que los estudiantes de salud presentan un perfil conformado por un predominio de las dimensiones conformidad, seguridad, realización, tradición y benevolencia. Se ha planteado, que estas dimensiones se agrupan en valores que regulan las relaciones con otros y los efectos sobre ellas, es decir centrados en lo social: universalismo, benevolencia, tradición conformidad y seguridad ${ }^{(20)}$. Esto coincidiría con el rol de promotor de salud y bienestar humano que se espera desempeñen los trabajadores de la salud. Aunque no puede afirmarse que este resultado es satisfactorio y esperado, pues se carece en la institución un estudio previo. Sin embargo, es un primer paso para contrastar en un segundo momento estos resultados con los valores declarados por la institución en el perfil de egreso de sus titulados y triangular con lo planteado por los académicos que están a cargo de la formación.

Si bien los estudiantes de las tres carreras están ligadas al cuidadoy promoción de la salud de las personas, se observan diferencias en las dimensiones: universalismo, poder, autodirección, benevolencia, conformidad, tradición y realización, estas en favor de los/las estudiantes de enfermería, que en gran parte pone a lo social como centro $y$ en un segundo momento a la persona ${ }^{(8,20)}$. En general los estudiantes de kinesiología y enfermería tenderían a presentar un perfil más cercano entre sí. Debe dejarse en claro que sólo se están comparando perfiles valóricos de carreras ligadas al cuidado y promoción de la salud de las personas a salud. Es muy probable que estos perfiles sean más homogéneos si son comparados con perfiles de estudiantes de otras áreas disciplinares.

Respecto al instrumento, como ya se señaló en el apartado resultados, las dimensiones hedonismo, seguridad y tradición presentan un nivel de confiabilidad inferior a, 60 , valor que diferentes autores consideran como mínimo de referencia para considerar que un instrumento o escala es confiable. Sin embargo, dado que se trata de un estudio exploratorio, estos valores aún podrían ser utilizados para interpretar los resultados de cada factor del estudio. Se profundizará en un segundo estudio la dimensionalidad del instrumento para verificar la estructura de la escala en una muestra mayor y de diferentes áreas geográficas, a través de un análisis factorial exploratorio y confirmatorio y su respectivos cálculos de fiabilidad por dimensión.

Los resultados muestran un perfil valórico de los estudiantes de salud, que debe ser tomado en cuenta como parte de su perfil de ingreso. El siguiente paso debería ser la comparación de este perfil resultante con lo que cada disciplina ha denominado el perfil de egreso de sus titulados, el que también posee explícita o implícitamente valores asociados.
El desarrollo de estudios para establecer estos perfiles estudiantiles en primer año, especialmente del area de salud permite reconocer la brecha que el curriculum formativo debe seguir para lograr un egresado con la formación disciplinar, científica y valórica que cada profesión ha establecido como propias, con el objetivo de promover el fortalecimiento o el desarrollo de estas dimensiones que cada carrera reconoce como importante para el desempeño profesión. Se observa además que con independencia que cada disciplina trabaje atendiendo la salud humana, existen diferencias en los perfiles valóricos declarados por los estudiantes y se esperaría lo mismo de los perfiles propios de cada profesión.

Finalmente, es importante evidenciar en los futuros profesionales de salud cómo se van configurando estos valores y cómo la formación profesional es capaz de incidir en sus valores personales y sociales con los que estos estudiantes acceden a la educación superior. El mapeo de éstos valores permitirá extrapolar que en el futuro laboral, los estudiantes tomen decisiones guiadas por los referentes éticos, principios y valores propios de la profesión, siendo esto un predictor significativo de la calidad de los cuidados que brindarán, de la satisfacción de los pacientes/usuarios que atiendan y de los propios profesionales al ejercer cotidianamente su profesión.

En conclusión, se puede señalar que los estudiantes de salud que participan del estudio conforman un perfil valórico en el que destacan las dimensiones conformidad, seguridad, realización, tradición y benevolencia de la escala de Schwartz. Se logran identificar diferencias en los perfiles de los estudiantes de acuerdo a la carrera estudiada.

\section{REFERENCIAS BIBLIOGRÁFICAS}

1. Servicio de información de Educación Superior, SIES. Panorama de la Educación Superior en Chile 2014. División de Educación Superior, Ministerio de Educación; 2014.

2. Leyton D, Vasquez A, Fuenzalida V. La experiencia de estudiantes de contextos vulnerables en diferentes Instituciones de Educación Superior Universitaria (IESU): Resultados de investigación. Calidad en la educación. 2012; 37: 61-97.

3. Ríos E, Moncada L, Llanos G, Santana R, Salinas H. Perfil psicológico de los estudiantes de 1 er año de enfermería: estudio preliminar. Ciencia y enfermería. 2009; 15(1): 99108.

4. Copparini N, Adorno H, Aguadé F, Arguello M, Báez L, Balbuena $S$, et al. Perfiles de personalidad de estudiantes de psicología de universidad privada comparados en similitud y congruencia. Eureka. 2011; 8 (1): 68-79.

5. Véliz-Burgos A, Urquijo P. Niveles de autoconcepto, autoeficacia académica y bienestar psicológico en estudiantes universitarios de la ciudad de Temuco. Salud \& Sociedad: investigaciones de la salud y pasicologia social. 2012; 3 (2): $131-50$.

6. Sobrinho J. Universidad, conocimiento y construcción de un mundo nuevo. InterCambios: Dilemas y Transiciones de la Educación Superior. 2014; 1(1): 10-9.

7. Schwartz S, Bardi A. Values hierarchies across cultures: Taking a similarities perspective. Journal of Cross-Cultural Psychology. 2001; 32(3): 268-90.

8. Schwartz, S. Universals in the content and structure of values: Theoretical advances and empirical test in 20 countries. Advances in experimental social psychology. 1992; 25: 1-65 
9. Delfino G, Zubieta E. Valores y política. Análisis del perfil axiológico de los estudiantes universitarios de la ciudad de Buenos Aires. Interdisciplinaria. 2011; 28(1); 93-114.

10. Navarro-Saldaña G, Pérez-Villalobos C, González-Cid A, MoraMardones 0 , Jiménez-Espinoza J. Valores en profesores y participación de los apoderados en el proceso de enseñanzaaprendizaje. Revista Latinoamericana de Psicología. 2007; 39(3): 579-92

11. Barría V, Bravo $B$, Cortés $R$, Vera $P$, Yévenes $M$, Zurita $C$. Estudio descriptivo exploratorio de la formación valórica en una muestra de estudiantes universitarios, según la teoría psicológica de los valores humanos de Shalom Schwartz (Seminario para optar al grado de Licenciado en psicología, Universidad de Concepción). Chile: Universidad de Concepción. 1999.

12. Carrasco E, Osses S. Transformaciones del perfil valorativo en estudiantes de la carrera de obstetricia y puericultura de la facultad de medicina de la Universidad de la Frontera durante su trayectoria académica. Estudios Pedagógicos. 2008; 34(2): 45-63.

13. García N. La función tutorial de la Universidad en el contexto actual de la Educación Superior. Revista Interuniversitaria de Formación del Profesorado. 2008; 22(1): 21-48.

14. Saíz J. Valores en estudiantes universitarios mapuches: Una visión transcultural de su contenido, estructura y jerarquía. [tesis doctoral]. Chile: Escuela de Psicología, Universidad de Chile; 2003.

15. Schwart, S. ¿Existen aspectos universales en la estructura y contenido de los valores humanos?. En M. Ros \& V. Gouveia (Eds.), Psicología social de los valores humanos. Madrid: Biblioteca Nueva. 2001: 53-76.

16. E. Emanuel. ¿Qué hace que la investigación clínica sea ética? Siete requisitos básicos, en $\mathrm{A}$. Pellegrini Filho y R. Macklin: Investigación en sujetos humanos: Experiencia Internacional. Programa Regional de Bioética. División de Salud y Desarrollo Humano. Organización Panamericana de la Salud/Organización Mundial de la Salud. Serie Publicaciones. 1999: 43-44.
17. Nunnally J, Bernstein I. Teoría Psicométrica. México, D.F: McGraw-Hill Latinomericana; 1995.

18. George D, Mallery P. SPSS for Windows step by step: A simple guide and reference. 11.0 update (4th ed.). Boston: Allyn \& Bacon; 2003.

19. Loewnthal K. An introduction to psychological tests and scales. London: UCL Press; 1996.

20. Fontaine JR, Poortinga YH, Delbeke L, Schwartz SH. Structural equivalence of the values domain across cultures. Distinguishing sampling fluctuations from meaningful variation. Journal of Cross-Cultural Psychology. 2008; 39 (4): 345-65.

Fuentes de financiamiento:

Este artículo ha sido financiado por los autores.

\section{Conflictos de interés:}

Los autores declaran no tener ningún conflicto de interés.

\section{Correspondencia:}

Alex Véliz Burgos

Dirección: Chinquihue Km6. Puerto Montt, Chile.

Teléfono: 0652322393

Correo electrónico: alex.veliz@ulagos.cl

Recibido: 07 de diciembre de 2016 Aprobado: 21 de abril de 2017 